\title{
Degradation Study of Metronidazole in Active and Different Formulation by UV Spectroscopy
}

\section{Safila Naveed*, Nimra Waheed and Safeena Nazeer}

Faculty of Pharmacy, Jinnah University for Women, Karachi, Pakistan

\begin{abstract}
Metronidazole (MTZ) is a 5-nitroimidazole semi-synthetic compound. MTZ is antiamoebic, anti protozoal and antibacterial, anti parasitic and anti trichomonal agents. It has wide range of activity against anaerobic bacteria as gram negative, gram positive bacilli and cocci, certain capnophilic organisms, protozoa and parasites. It is indicated in infections caused by Helicobacter pylori, acne rosacea, anaerobic infections, parasitic infections, pseudomembranous colitis and Crohn's disease. ICH guidelines states the parameters which causes forced degradation of a drug product includes Time, Temperature and/ or with humidity, Acid/base Stress testing, photo degradation and $\mathrm{pH}$ variation (high and low). Ultraviolet-visible spectroscopic process was developed to analyze and calculate the amount of drug in the presence of degradation products. According to the USP, the official assay limit of the content should NLT $90 \%$ and NMT $110 \%$ of labeled amount of metronidazole. When active of metronidazole and different brands (FGL, MNE, KNT) exposed to acidic medium of $1 \mathrm{~N} \mathrm{HCI} \mathrm{FGL,} \mathrm{MNE} \mathrm{and} \mathrm{KNT} \mathrm{show} \mathrm{heavy} \mathrm{degradation} \mathrm{whereas} \mathrm{as} \mathrm{active} \mathrm{MTZb}$ didn't show any degradation. When active of metronidazole and FGL, MNE, KNT exposed to basic medium i.e. 1N $\mathrm{NaOH}, \mathrm{FGL}$ show moderate degradation, MNE and KNT shows slight degradation whereas as active didn't show any degradation. FGL, MNE, KNT and ND MTZb when subjected to heat none of them show degradation. When MTZb, FGL, MNE and KNT subjected to UV light, MTZb exhibit heavy degradation whereas FGL, MNE and KNT exhibit no degradation. UV-Vis spectroscopy is a simple, rapid, cost effective, useful and fruitful method for determination of MTZ in their active and pharmaceutical dosage form (tablets) of different brands.
\end{abstract}

Keywords: Metronidazole; Degradation; Active; Formulation

\section{Introduction}

Semi synthetic 5-nitroimidazole compound for example Metronidazole (MTZ) was first time found in 1959 in France at Rhone Poulanc Research Laboratories and presented for the Trichomonas vaginalis infections treatment clinical medicine. It is antiamoebic, antiprotozoal and antibacterial, antiparasitic and antitrichomonal agents. MTZ when use as bactericidal agent it work at low concentration. Its range of activity includes almost all anaerobic (but not aerobic) bacteria such as gram-ve, gram +ve (Clostridium difficile, Gardnerella vaginalis), bacilli and cocci, certain capnophilic organisms protozoa such as Trichomonas vaginalis, Entamoeba histolytica, and Giardia lamblia. it has been clinically use for several years and is a drug of first choice for treatment of Helicobacter pylori, acne rosacea, anaerobic infections, parasitic infections, pseudomembranous colitis and Crohn's disease. For biological activation of metronidazole the 5-nitro group on the ring of imidazole to be reduced. One kind of data to sustain this analysis is the relationship between the electron affinity of the nitro group in a sequence of nitroimidazoles and their mutagenic, radiation-sensitizing and cytotoxic potencies. There are many other biological effects that also seem to depend on activity of nitro reductase. Consequently, the sensitivity of nitro reductase action to oxygen can elucidate why metronidazole has less bactericidal activity for aerobic bacteria as compare to the activity for anaerobic bacteria and less cytotoxic effect for oxygenated tissue culture cells as compared to the cells that grown under the condition of hypoxia [1-7]. Metronidazole is white or creamy-white crystalline powder chemically called as of 2-(2-methyl5-Nitro-1H-imidazol-1-yl) ethanol having a chemical structure shown below (Figure 1)

It is bitter slightly saline taste with a minor odor and when expose to light it darkens. It is almost soluble in water $(1 \mathrm{~g}$ in $100 \mathrm{ml})$, alcohol $(1 \mathrm{~g}$ in $200 \mathrm{ml})$, chloroform $(1 \mathrm{~g}$ in $250 \mathrm{ml})$ and somewhat soluble in ether. MTZ is available in the form of tablet, infusion and suppositories.
Film coated tablets are available with the strength of $400-800 \mathrm{mg}$. It is readily absorbed after oral administration from the Gastro-intestinal (GI) tract and extensively distributed into all body tissues and fluids. It is metabolized by liver through conjugation and oxidation with process glucuronic acid into its 2 metabolites i.e. acid oxidation product and a glucuronide. It eliminates after half- life of seven to eight hours and excretes one-half of MTZ in urine [8-12].

British Pharmacopoeia (BP), United States Pharmacopoeia (USP) and Indian Pharmacopoeia (IP) have determined many official methods for assay of MTZ. British Pharmacopoeia (BP) explains nonaqueous $(\mathrm{N}-\mathrm{A})$ and potentiometric methods using perchloric acid (PCA) as titrant whereas USP illustrates high performance liquid chromatography and (N-A) titration methods while the IP describes the malachite green as indicator and (N-A) titration method using

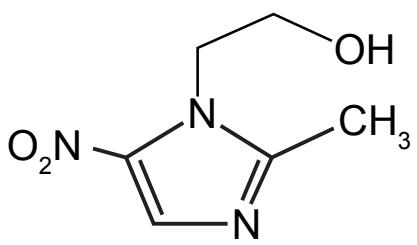

Figure 1: Structure of Metronidazole.

*Corresponding author: Safila Naveed, Faculty of Pharmacy, Jinnah University for
Women, Karachi, Pakistan, E-mail: safila117@yahoo.com; safila117@gmail.com Received June 25, 2014; Accepted July 21, 2014; Published July 29, 2014

Citation: Naveed S, Waheed N, Nazeer S (2014) Degradation Study of Metronidazole in Active and Different Formulation by UV Spectroscopy. J Bioequiv Availab 6: 124-127. doi:10.4172/jbb.1000191

Copyright: (c) 2014 Naveed S. This is an open-access article distributed under the terms of the Creative Commons Attribution License, which permits unrestricted use, distribution, and reproduction in any medium, provided the original author and source are credited. 
PCA as titrant for the assay of MTZ. Several other methods have been accounted for e.g. spectrophotometry (mostly in visible region) and polarography [13]. MTZ can be determined alone or in addition to its mixture with other drugs or in the presence of its degradation product or in the presence of its metabolites with the help of HPLC methods, NMR spectrometry, gas chromatography, voltammetry and short-wave length NIR spectroscopy. Derivative spectrophotometry was used for the first time for the determination of MTZ in mixture with ciprofloxacin [14].

International Conference of Harmonization (ICH) guideline specifies that active pharmaceutical ingredient (API) is focused to numerous forced degradation conditions including basic, acidic and photo degradation [15]. Initial improvement process, forced degradation activities must be executed to ensure that the process is selective to save lot of time, effort, money and to find out the responsible conditions for drug degradation [16]. Forced degradation is capable of signifying that the selected method is indicating the stability of API i.e. an identifying method that used to detect the intensification of the degradation product and the successive loss of active components [17].

In the present study Ultraviolet-visible (UV-Vis) spectroscopy is used for the analysis of forced degradation of Metronidazole. UV-Vis spectroscopy is a simple, rapid, cost effective useful and fruitful method for determination of MTZ in their active and pharmaceutical dosage form (tablets) of different brands. Furthermore, they do not need costly instrumentation required for published HPLC methods.

\section{Factors affecting forced degradation}

The characteristic factors which affect API for forced degradation are Time, Temperature and/ or with humidity, Acid/base Stress testing, photo degradation and $\mathrm{pH}$ variation (high and low).

Thermal and/or humidity stress testing: This test is initiated as the drug substance is place in thermal/humidity conditions for extended period of time which consequently causes the active substance to forcefully degrade to its primary constituents.

Acid/base stress testing: This test is used to evaluate the forced degradation of an API which involves exposure of a drug substance in basic or acidic environment for longer period of time causes degradation to its primary constituents. Acid/base hydrolysis take place in labile carbonyl functional groups for e.g. esters (lactones),amides (lactams), alcohols, carbamates, imides, imines and aryl amines.

Degradation by UV light: Several UV-unstable products have a major problem of Ultraviolet-visible degradation. UV-unstable products consist of synthetic and natural polymers which crack or disintegrate when exposed to constant sunlight. Continuous exposure is a more severe problem than discontinuous exposure because the attack is dependent on the extent and degree of exposure [16].

\section{Experimental}

Metronidazole: For degradation study and assay we use different brands of metronidazole tablets $400 \mathrm{mg}$ i.e. Flagyl 'FGL' (multinational), Metrozine 'MNE' (local), Klint 'KNT' (local) and the active metronidazole benzoate 'MTZb'.

Instruments: Weighing Balance (Item PA214C) 'Pioneer OHAIUS', Spectrophotometer with a quartz cuvette (T80 ultravioletvisible spectrometer) 'PG Instrument', Ultraviolet Lamp (Serial no. N 045571, Power of 8N, LF-204.LS) '4W-254 and 365 nm' and Water Bath 'HH-4' having Digital and constant temperature tank
Reagents: Reagents were used $1 \mathrm{~N} \mathrm{NaOH}$ (sodium hydroxide), $1 \mathrm{~N}$ $\mathrm{HCl}$ (hydrochloric acid) and DI (de-ionized) water. DI water was double distilled, de-ionized and filtered). All of the reagents used were of analytical grade.

Glass wares: Glass wares were of Pyrex type which includes beakers, funnel, pipette, Measuring cylinder, stirrer and volumetric flask. All the glasswares were initially washed with chromic acid afterward with water and lastly rinsed with freshly prepared double distilled or DI water.

\section{Preparation of $0.1 \mathrm{~N}$ hydrochloric acid}

For the preparation of $\mathrm{HCl}$, took $8.3 \mathrm{ml}$ analytical grade $\mathrm{HCl}$ of $37 \%$ purity and 12 normality in a volumetric flask and the make up the final volume with DI water up to the mark of flask.

\section{Preparation of $0.1 \mathrm{~N}$ sodium hydroxide}

For the preparation of $\mathrm{NaOH}$, weigh four grams of $\mathrm{NaOH}$. Firstly we transfer $\mathrm{NaOH}$ in $100 \mathrm{ml}$ volumetric flask and dissolve it in small quantity of DI water and finally make up the volume with DI water up to mark of the flask.

\section{Preparation of metronidazole solution}

Individually weigh the metronidazole tablet of each brand on the weighing balance. Crush and mix the tablets in mortar pestle. Accurately weigh the powders for making primary solutions equivalent to $10 \mathrm{mg}$ of metronidazole i.e. $(0.0157 \mathrm{gm})$ while directly weigh the active (metronidazole benzoate) $0.0100 \mathrm{gm}$. Transfer these weighed samples in 4 separate volumetric flasks of $100 \mathrm{ml}$. Initially, powder was dissolve in small quantity of water than shaking was carried out to completely dissolve it and finally make up the volume with water to $100 \mathrm{ml}$ respectively for each sample while the active was initially dissolve in acetone than finally make up the volume with water to $100 \mathrm{ml}$. to determine the absorbance spectrophotometer was used in which solutions (desired concentration) of $100 \mathrm{ppm}$ were transferred to cuvette separately and absorbance were determined at max $278 \mathrm{~nm}$.

\section{Method of degradation studies}

For Acid: Take $5 \mathrm{ml}$ of $100 \mathrm{ppm}$ solution of MTZb (active), FGL (multinational), MNE (local) and KNT (local) in four different test tubes to assess the effect of acid ( $\mathrm{HCl})$. In same test tube, add $5 \mathrm{ml}$ of $1 \mathrm{~N}$ hydrochloric acid and left then for the 30 minutes. Afterwards the absorbance of the solutions were analyze separately at wavelength max $278 \mathrm{~nm}$.

For Base: Take $5 \mathrm{ml}$ of $100 \mathrm{ppm}$ solution of MTZb (active), FGL (multinational), MNE (local) and KNT (local) in four different test tubes to assess the effect of base $(\mathrm{NaOH})$. In same test tube, than add $5 \mathrm{ml}$ of $1 \mathrm{~N}$ sodium hydroxide and left then for the 30 minutes. Afterwards the absorbance of the solutions were analyze separately at wavelength $\max 278 \mathrm{~nm}$.

For UV light: Take $5 \mathrm{ml}$ of $100 \mathrm{ppm}$ solution of MTZb (active), FGL (multinational), MNE (local) and KNT (local) in four different test tubes to assess the effect of UV (ultraviolet) light. In same test tube, than add $5 \mathrm{ml}$ of DI water and left these test tubes in UV light of 320 $\mathrm{nm}$ for the 30 minutes. Afterwards the absorbance of the solutions were analyze separately at wavelength $\max 278 \mathrm{~nm}$.

For Heat: Take $5 \mathrm{ml}$ of $100 \mathrm{ppm}$ solution of MTZb (active), FGL (multinational), MNE (local) and KNT (local) in four different test tubes to assess the effect of heat. In same test tube, than add $5 \mathrm{ml}$ of DI 
Citation: Naveed S, Waheed N, Nazeer S (2014) Degradation Study of Metronidazole in Active and Different Formulation by UV Spectroscopy. J Bioequiv Availab 6: 124-127. doi:10.4172/jbb.1000191

water and left these test tubes in water bath for the 30 minutes at $50^{\circ} \mathrm{C}$. Afterwards the absorbance of the solutions were analyze separately at wavelength $\max 278 \mathrm{~nm}$.

\section{Result}

We study degradation parameters on active of metronidazole i.e. metronidazole benzoate and 3 brands of it i.e. Flagyl (FGL), Metrozine (MNE) and Klint (KNT). (Tables 1 and 2, Figures 2 and 3) When MTZb, FGL, MNE and KNT are subjected to $1 \mathrm{~N} \mathrm{HCl}$, the MTZb do not show any changes whereas all three brands i.e. FGL, MNE and KNT showed increased availability (234\%, 243.27\%, 238.27\%) respectively. In the same way when MTZb, FGL, MNE and KNT are subjected to $1 \mathrm{~N} \mathrm{NaOH}$, the MTZb do not showed any changes whereas all three brands i.e. FGL, MNE and KNT showed increased availability $(154.27 \%, 131.50 \%, 137.23 \%)$ respectively. No changes were seen when MTZb, FGL, MNE and KNT are exposed to heat for 30 minutes. When
MTZb, FGL, MNE and KNT are exposed to U.V light, the MTZb shows decreased availability (25.68\%) whereas all three brands do not show any changes.

From our results we can conclude that MTZb when introduced in acidic medium i.e. $1 \mathrm{~N} \mathrm{HCl}$ do not degrade whereas $\mathrm{MNE}$ degrades to a larger extend (243.27\%). MTZb do not degrades when subjected to basic medium i.e. $1 \mathrm{~N} \mathrm{NaOH}$ and FGL degrades highly (154.27\%) among the 3 brands of metronidazole (FGL, MNE and KNT). MTZb, FGL, MNE and KNT do not degrade when heated for $30 \mathrm{~min}$. MTZb degrades when expose to U.V light whereas FGL, MNE and KNT when expose to U.V light. They are in the official limits i.e. $90-110 \%$ of the drug should be available in \% assay.

\section{Conclusion}

According to United States Pharmacopoeia (USP) specification,

\begin{tabular}{|c|c|c|c|c|c|c|c|c|c|c|c|c|c|c|c|c|}
\hline \multirow{2}{*}{$\begin{array}{l}\text { Degradation } \\
\text { Parameters }\end{array}$} & \multicolumn{4}{|c|}{ MTZb } & \multicolumn{4}{|c|}{ FGL } & \multicolumn{4}{|c|}{ MNE } & \multicolumn{4}{|c|}{ KNT } \\
\hline & 1 & 2 & 3 & Avg. & 1 & 2 & 3 & Avg. & 1 & 2 & 3 & Avg. & 1 & 2 & 3 & Avg. \\
\hline before & 2.48 & 2.49 & 2.49 & 2.487 & 0.62 & 0.62 & 0.62 & 0.62 & 0.67 & 0.67 & 0.67 & 0.67 & 0.67 & 0.67 & 0.67 & 0.67 \\
\hline after acid & 2.45 & 2.47 & 2.46 & 2.46 & 1.45 & 1.45 & 1.45 & 1.45 & 1.63 & 1.63 & 1.63 & 1.63 & 1.59 & 1.59 & 1.59 & 1.59 \\
\hline after base & 2.54 & 2.5 & 2.48 & 2.507 & 0.95 & 0.95 & 0.96 & 0.95 & 0.882 & 0.88 & 0.88 & 0.88 & 0.92 & 0.92 & 0.92 & 0.92 \\
\hline after heat & 2.7 & 2.73 & 2.75 & 2.727 & 0.64 & 0.64 & 0.64 & 0.64 & 0.71 & 0.71 & 0.71 & 0.71 & 0.67 & 0.67 & 0.67 & 0.667 \\
\hline after U.V & 0.63 & 0.64 & 0.63 & 0.633 & 0.63 & 0.63 & 0.63 & 0.63 & 0.68 & 0.69 & 0.69 & 0.69 & 0.67 & 0.67 & 0.67 & 0.667 \\
\hline
\end{tabular}

Table 1: Absorbance of Active and Different Brands.

\begin{tabular}{|c|c|c|c|c|c|c|c|c|}
\hline \multirow{2}{*}{$\begin{array}{c}\text { Degradation } \\
\text { Parameters }\end{array}$} & \multicolumn{4}{|c|}{ MTZb } & \multicolumn{4}{|c|}{ FGL } \\
\hline & 1 & 2 & 3 & Avg. & 1 & 2 & 3 & Avg. \\
\hline before & $99.73 \%$ & $100 \%$ & $100 \%$ & $99.98 \%$ & $100 \%$ & $100 \%$ & $100 \%$ & $100 \%$ \\
\hline after acid & $98.70 \%$ & $99.60 \%$ & $99 \%$ & $99.17 \%$ & $234 \%$ & $234 \%$ & $234 \%$ & $234 \%$ \\
\hline after base & $102.18 \%$ & $100.57 \%$ & $99.73 \%$ & $100.83 \%$ & $153.70 \%$ & $154.30 \%$ & $154.80 \%$ & $154.27 \%$ \\
\hline after heat & $108.80 \%$ & $109.90 \%$ & $110.50 \%$ & $109.73 \%$ & $103.50 \%$ & $103.50 \%$ & $103.50 \%$ & $103.50 \%$ \\
\hline \multirow[t]{3}{*}{ after U.V } & $25.30 \%$ & $26.05 \%$ & $25.69 \%$ & $25.68 \%$ & $101.90 \%$ & $101.90 \%$ & $102.10 \%$ & $101.97 \%$ \\
\hline & \multicolumn{4}{|c|}{ MNE } & \multicolumn{4}{|c|}{ KNT } \\
\hline & 1 & 2 & 3 & Avg. & 1 & 2 & 3 & Avg. \\
\hline before & $100 \%$ & $100 \%$ & $100 \%$ & $100 \%$ & $100 \%$ & $100 \%$ & $100 \%$ & $100 \%$ \\
\hline after acid & $243.20 \%$ & $243.40 \%$ & $243.20 \%$ & $243.27 \%$ & $238.00 \%$ & $238.30 \%$ & $238.50 \%$ & $238.27 \%$ \\
\hline after base & $131.60 \%$ & $131.30 \%$ & $131.60 \%$ & $131.50 \%$ & $137.30 \%$ & $137.30 \%$ & $137.10 \%$ & $137.23 \%$ \\
\hline after heat & $105.30 \%$ & $105.20 \%$ & $105.30 \%$ & $105.27 \%$ & $99.40 \%$ & $99.50 \%$ & $99.55 \%$ & $99.48 \%$ \\
\hline after U.V & $102.80 \%$ & $102.90 \%$ & $102.90 \%$ & $102.87 \%$ & $99.55 \%$ & $99.70 \%$ & $99.55 \%$ & $99.60 \%$ \\
\hline
\end{tabular}

Table 2: Degradation Pattern in Percentage of Metronidazole (Active and its Brands).

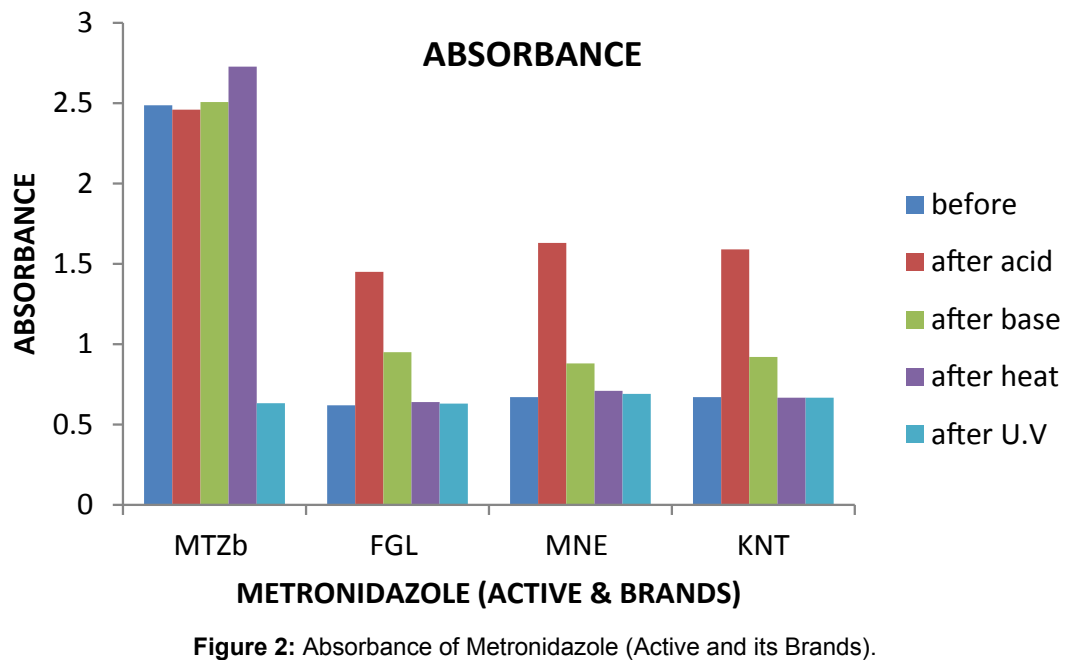


Citation: Naveed S, Waheed N, Nazeer S (2014) Degradation Study of Metronidazole in Active and Different Formulation by UV Spectroscopy. J Bioequiv Availab 6: 124-127. doi:10.4172/jbb.1000191

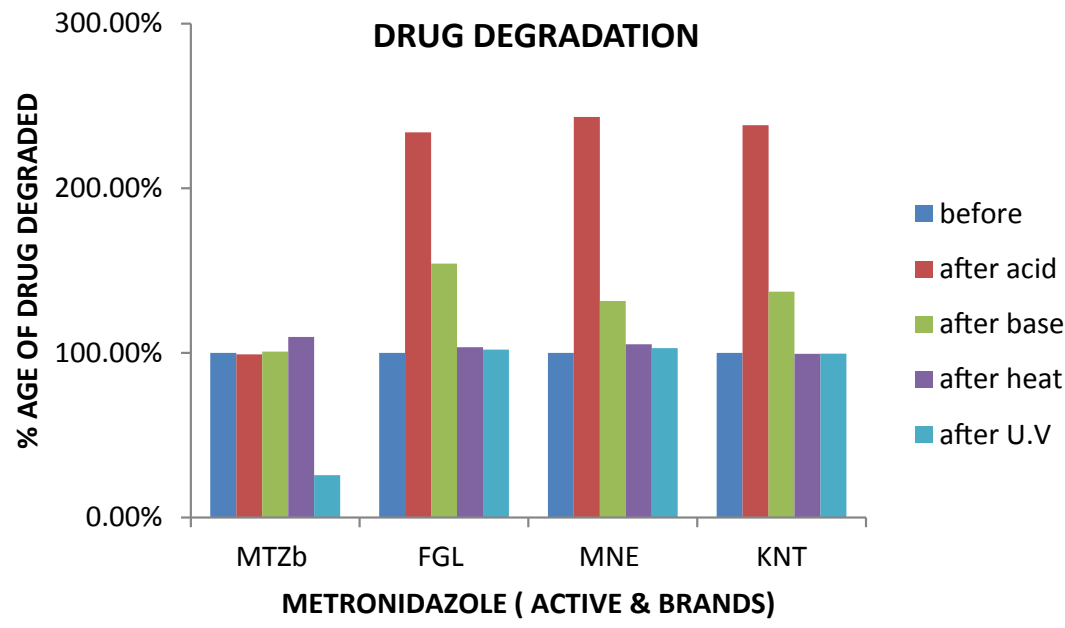

Figure 3: Degradation Pattern.

the official assay limit of the content should NLT $90 \%$ and NMT $110 \%$ of labeled amount. From our performance we can conclude that formulations of metronidazole degrades most in acidic medium whereas slight degradation occurs in basic medium but its active do not degrades in both acidic and basic medium. Furthermore, moderate degradation in active occurs when expose to U.V light whereas heat do not degrade either active or any of the formulation of metronidazole.

\section{References}

1. Agudelo $M$, Vesga $O$ (2012) Therapeutic equivalence requires pharmaceutical, pharmacokinetic, and pharmacodynamic identities: true bioequivalence of a generic product of intravenous metronidazole. Antimicrob Agents chemother 56: 2659-2665.

2. Tally FP, Goldin BR, Sullivan N, Johnston J, Gorbach SL (1978) Antimicrobial activity of metronidazole in anaerobic bacteria. Antimicrob Agents Chemother 13: $460-465$.

3. Siddappa K, Mallikarjun M, Reddy PT, Tambe M (2008) Spectrophotometric determination of metronidazole through Schiff's base system using vanillin and PDAB reagents in pharmaceutical preparations. Eclética Química.

4. Faisal A, Sultana T, Didaruzzaman Sohel Md, Helal Uddin Sumon Md, Hassan Kawsar Md, et al (2014) In vitro dissolution pattern of metronidazole film coated tablet in presence of fruit juice. American journal of pharmacological sciences 2: $32-36$

5. Al-sabea N (2008) Assay of metronidazole from different manufacturing sources in Iraqi markets. AJPS 1: 16-25

6. Lockerby DL, Rabin HR, Bryan LE, Laishley EJ (1984) Ferredoxin-linked reduction of metronidazole in clostridium pasteurianum. Antimicrob Agents Chemother 25: 665-669.

7. Chrystal EJ, Koch RL, McLafferty MA, Goldman P (1980) Relationship between Metronidazole Metabolism and Bactericidal Activity. Antimicrob Agents Chemother 18: 566-573.

8. Alhalabi Z, Al-Khayat MA, Haidar S (2012) Separation and assay of antiprotozoal imidazole derivatives (metronidazole, tinidazole and secnidazole) by RP-HPLC. International Journal of Pharmaceutical Sciences Review and Research 13: 12-18.
9. Clarcks (2001) Isolation \& Identification of Drugs

10. Budhiraja RD (2009) Elementary Pharmacology \& Toxicology, Popular Prakashan, Mumbai p432-433.

11. Harrison's principles of internal medicine. International edition-cd-rom.

12. (1982) Martindile: the extra pharmacopoeia, from royal pharmaceutical Society of Great Britain.

13. Thulasamma P and Venkateswarlu P (2009) Spectrophotometric Method for the Determination of Metronidazole in Pharmaceutical Pure and Dosage Forms. Rasayan journal 865-868.

14. Mohamed R. El-Ghobashy, Nisreen F. Abo-Talib (2010) Spectrophotometric methods for the simultaneous determination of binary mixture of metronidazole and diloxanidefuroate without prior separation. Journal of Advanced Research p323-329.

15. Food and Drug Administration, HHS (2003) International Conference on Harmonisation; Stability Data Package for Registration Applications in Climatic Zones III and IV; Stability Testing of New Drug Substances and Products: availability. Notice. See comment in PubMed Commons below Fed Regist 68: 65717-65718.

16. Naveed S, Shafiq A, Khan M, Jamal M, Zafar H, et al. (2014) Degradation Study of Available Brands of Metformin in Karachi Using UV Spectrophotometer. J Diabetes Metab 5: 328

17. Kishore kumarhotha (2013) Forced degradation studies: practical approachoverview of regulatory guidance and literature for the drug products and drug substances. 